\title{
A Guideline to Select Control Charts For Multiple Stream Processes Control
}

\author{
Prapasak Jirasettapong* and Napassavong Rojanarowan \\ Department of Industrial Engineering, \\ Faculty of Engineering, Chulalongkorn University \\ Bangkok, Thailand 10330 \\ E-mail: prapasakj@yahoo.com* and napassavong.o@ chula.ac.th
}

\begin{abstract}
Multiple-stream processes (MSP) can usually be found in a factory. MSP control chart is one of the tools used to control these processes. It is required that MSP control charts are selected appropriately based on streams characteristics, in order to minimize false decision on process status and irrelevant action on the process. This paper presents the guideline and a case study of selecting MSP control charts by considering the major factors, which are correlation among streams, number of streams, limitation to use one chart per stream, difference among streams average, and shift size of streams average to be detected.
\end{abstract}

Keywords: Control chart selection, control charts for multiple-stream processes, multiple-stream processes control.

ENGINEERING JOURNAL Volume 15 Issue 3

Received 20 February 2011

Accepted 19 March 2011

Published 1 July 2011

Online at http://www.ej.eng.chula.ac.th/eng/

DOI:10.4186/ej.2011.15.3.1 


\section{Introduction}

In multiple stream processes where the machine has several modules, each module is capable for producing the output simultaneously, e.g. a multi-slot testing machine or a multi-spindle screwfastening machine. The output from each test slot or each spindle is considered as output from a process stream. The possible guideline for using control chart in this kind of process is to use a single chart on (any) one stream or a single chart on all streams or one chart per stream.

Since Boyd [1] introduced the application of Group control chart in 1950, the subsequent articles almost stress on introducing new MSP control charts to resolve the drawbacks of preceding charts and to serve different situations of practical applications. Mortell and Runger [2] show disadvantages of the group control chart and present an alternative approach, the $\overline{\bar{X}}-R_{t}$ control charts. Grimshaw et al. [3] demonstrate that the $\mathrm{ARL}_{0}$ of group control chart is decreased when the number of streams increases. They also propose the new control limits for group control chart for maintaining $\mathrm{ARL}_{0}$ of group control chart at approximately 370. Meneces et al. [4] point out the drawbacks of group control chart and $\overline{\bar{X}}-R_{t}$ control charts and propose Shewart control chart for each stream. Liu et al. [5] introduce MSP control charts using more complex statistics that are F statistics and likelihood ratio. For process with large number of streams and sample collection from all streams are not possible and the shift size to be detected is moderate or large, Lanning et al. [6] present MSP control charts for this special case using fractional samples and adaptive sampling plan. While new MSP control charts continued to be developed, none have yet addressed how to select the MSP control charts. Each MSP control chart has advantages and drawbacks. Selecting MSP control charts that does not comply with streams characteristics will lead to wrong judgment on process status and irrelevant action to the process. This paper presents a guideline to select control charts for multiple stream processes in order to select MSP control charts that correspond to streams characteristics and applicable to the practical operation. This paper excludes the complex MSP controls charts, the F test and a likelihood ratio test charts, the fractional samples and adaptive sampling plan chart from the guideline because they are difficult to design, implement, and interpret by practitioners and operators. In section II, the advantages and drawbacks of MSP control charts included in the consideration are discussed. Section III presents a guideline to select control charts for multiple stream processes control followed by a case study in section IV and conclusion in section V.

\section{Multiple stream process control charts}

\subsection{Single control chart on (any) one stream}

In the case that the streams are nearly perfectly correlated, using a popular control chart such as $\bar{X}-R$, $\bar{X}-s$ or EWMA on any one stream is applicable [7] because when one stream is out of control, the others tend to be out of control also. Nonetheless, in general, correlation is usually moderate so using single control chart on one stream cannot represent all streams status because if one stream is out of control, the others may or may not be out of control. In this case, one chart per stream should be used to answer the question "Do some of streams or all streams are out of control?" Then, the action should correspond to the out of control condition. That is, if all streams are out of control, action should be focused on parameters that are common to all streams. However, if some streams are out of control the action should only limit to the streams that are out of control.

\subsection{Group control chart}

Group Control Chart or GCC is the MSP control chart that is used only a single chart on all streams in order to reduce the number of charts required to monitor the processes. This control chart was introduces by Boyd in 1950 and generally mentioned in the papers of MSP control charts. GCC comprises of group $\bar{X}$ chart and group $R$ chart. The calculations of the center line and control limits use the same equations and constants as $\bar{X}-R$ chart. The largest and smallest of $\bar{X}$ and $R$ are plotted into group $\bar{X}$ chart and group $R$ chart respectively. The number of streams correspond to the plotted points are also specified in the charts to facilitate the streams identification when the process out of 
control. If largest and smallest points are inside the control limits, all others points are also lie inside the limits indicating that all streams are in control. When a point lies outside a control limit, at least one stream is out of control. If both largest and smallest points are out of control in the same side of a control limit, it implies that every stream is out of control. Besides the consideration of the point exceeds the limits, if a stream consistently yields the largest or smallest value several times in a row, it implies that this stream is out of control as well.

Although using GCC reduces a number of control charts required in MSP processes and provides understanding for operator as it is similar to the $\bar{X}-R$ chart, GCC also has several weaknesses as follows:

1) The control limits of control charts, in general, are specified at \pm 3 sigma. This makes false alarm event happen every 370 samples or yields average run length in case of process is in control or $\mathrm{ARL}_{0}$ approximately 370. $\mathrm{ARL}_{0}$ of 370 is commonly used in control chart design. Although desired $\mathrm{ARL}_{0}$ can be achieved by increasing or decreasing the control limit from \pm 3 sigma limit, Grimshaw et al. [3] have demonstrated that even though control limits are maintained at \pm 3 sigma, $\mathrm{ARL}_{0}$ of GCC is decreased when the number of streams increase as shown in Eq. (1).

$$
\mathrm{ARL}_{0}=\frac{1}{1-(0.9973)^{k}}
$$

where $k$ is the number of streams.

2) In case a particular stream is out-of-control by showing $r$ consecutive largest (or smallest) points on GCC which Mortell and Runger [2] refer to as the one-sided run scheme, the one-sided in-control average run length or ARL(1) can be calculated using Eq. (2) from Nelson [8]

$$
\operatorname{ARL}(1)_{0}=\frac{k^{r}-1}{k-1}
$$

where $k$ is the number of streams and $r$ is the number of consecutive times that a particular stream is the maximum (or minimum) value.

Based on Eq. (2), ARL(1) $)_{0}$ of GCC with 2-25 streams are shown in Table 1. It can be noticed that the one-sided in-control average run length or ARL $(1)_{0}$ is highly discrete. The discreteness of the incontrol ARL will be more substantial when the number of streams increases. In addition, at some number of streams, there are no schemes that yield ARL(1) close to 370. Choosing run scheme with the lower ARL results in too many false alarms. On the other hand, using the run scheme with the higher ARL results in many more samples to be taken before the special cause is detected. From Table 1, the highlighted ARL(1) $)_{0}$ show the cases where the ARL(1) deviate from common ARL at 370 by $\pm 10 \%$. The data in table 1 imply that if $\pm 10 \%$ deviations are required, the one-sided run scheme is only applicable to the process with $3,4,7,18$ and 19 streams. In other cases, the ARL $(1)_{0}$ deviate from common ARL at 370 for more than $\pm 10 \%$. 
Table 1. ARL(1) values for Group Control Chart with 2-25 streams.

\begin{tabular}{|c|c|c|c|c|c|c|c|c|}
\hline \multirow{2}{*}{$\begin{array}{l}\text { Number of } \\
\text { streams (k) }\end{array}$} & \multicolumn{8}{|c|}{ Consecutive points $(r)$} \\
\hline & 2 & 3 & 4 & 5 & 6 & 7 & 8 & 9 \\
\hline 2 & 3 & 7 & 15 & 31 & 63 & 127 & 255 & 511 \\
\hline 3 & 4 & 13 & 40 & 121 & 364 & 1093 & 3280 & 9841 \\
\hline 4 & 5 & 21 & 85 & 341 & 1365 & 5461 & 21845 & 87381 \\
\hline 5 & 6 & 31 & 156 & 781 & 3906 & 19531 & 97656 & \\
\hline 6 & 7 & 43 & 259 & 1555 & 9331 & 55987 & & \\
\hline 7 & 8 & 57 & 400 & 2801 & 19608 & & & \\
\hline 8 & 9 & 73 & 585 & 4681 & 37449 & & & \\
\hline 9 & 10 & 91 & 820 & 7381 & 66430 & & & \\
\hline 10 & 11 & 111 & 1111 & 11111 & & & & \\
\hline 11 & 12 & 133 & 1464 & 16105 & & & & \\
\hline 12 & 13 & 157 & 1885 & 22621 & & & & \\
\hline 13 & 14 & 183 & 2380 & 30941 & & & & \\
\hline 14 & 15 & 211 & 2955 & 41371 & & & & \\
\hline 15 & 16 & 241 & 3616 & 54241 & & & & \\
\hline 16 & 17 & 273 & 4369 & 69905 & & & & \\
\hline 17 & 18 & 307 & 5220 & 88741 & & & & \\
\hline 18 & 19 & 343 & 6175 & & & & & \\
\hline 19 & 20 & 381 & 7240 & & & & & \\
\hline 20 & 21 & 421 & 8421 & & & & & \\
\hline 21 & 22 & 463 & 9724 & & & & & \\
\hline 22 & 23 & 507 & 11155 & & & & & \\
\hline 23 & 24 & 553 & 12720 & & & & & \\
\hline 24 & 25 & 601 & 14425 & & & & & \\
\hline 25 & 26 & 651 & 16276 & & & & & \\
\hline
\end{tabular}

3) When consider the process out of control from a particular stream that yield either largest or smallest in consecutive points plotted on GCC or the two-sided run scheme, Mortell and Runger [2] demonstrates $\mathrm{ARL}_{0}$ in this case as $\operatorname{ARL}(2)_{0}$. Similar to $\operatorname{ARL}(1)_{0}$, the $\operatorname{ARL}(2)_{0}$ are more discrete as the number of streams increases.

4) In case the mean of each stream is significantly different, the false alarm of run scheme will be increased because the streams with largest or smallest mean have more opportunity to be plotted on the chart than others.

5) Using GCC, a single chart on all streams, provide less information than that of the charts that are separated on each stream. The less information may slow down the troubleshooting as more data may need to be collected and analyzed.

\subsection{Group Control Chart with Adjusted Control Limits}

To eliminate the weakness of GCC that the $\mathrm{ARL}_{0}$ is decreased when the number of streams increase, Grimshaw et al. [3] introduced the adjusted control limits which correspond to the number of streams for GCC in order to maintain $\mathrm{ARL}_{0}$ at 370 . The GCC with adjusted control limits are similar to the original GCC except the control limits are selected based on the subgroup size and number of streams.

\subsection{Mortell and Runger Multiple Stream Processes Control Charts}

Mortell and Runger [2] introduced MSP control charts that can use a single chart on all streams. That is the $\overline{\bar{X}}-R_{t}$ control charts. The $\overline{\bar{X}}$ chart uses the grand average of all streams to detect the special cause 
that makes all streams out of control. For the $\overline{\bar{X}}-R_{t}$ chart, the average range of all streams will be used to detect the special cause that makes one or several streams out of control. The statistic $R_{t}$ can be plotted into 3 types of control charts that are Shewhart control chart, CUSUM and EWMA

Mortel and Runger [2] compared the sensitivity of various MSP control charts in the case that one stream is out of control by comparing the ARL of each chart. Figure 1(a), 1(b), 1(c), and 1(d) shows the comparisons of the ARLs for the different MSP control charts for 2, 3, 5, and 10 streams, respectively. These charts are denoted as CUSA, CUSR, EWM1, EWM3, EWM8, RUNS, and SHEW corresponding to a CUSUM chart using the maximum residual as the control variable, a CUSUM chart using $R_{t}$ as the control variable, an EWMA chart based on $R_{t}$ with the weighting factor equal to 0.1 , 0.3 and 0.8 , GCC with run scheme, and a Shewhart chart using $R_{t}$ as the control variable respectively. The plot $u=0.0$ at the top of each graph is zero mean shift which provide the in-control ARL.

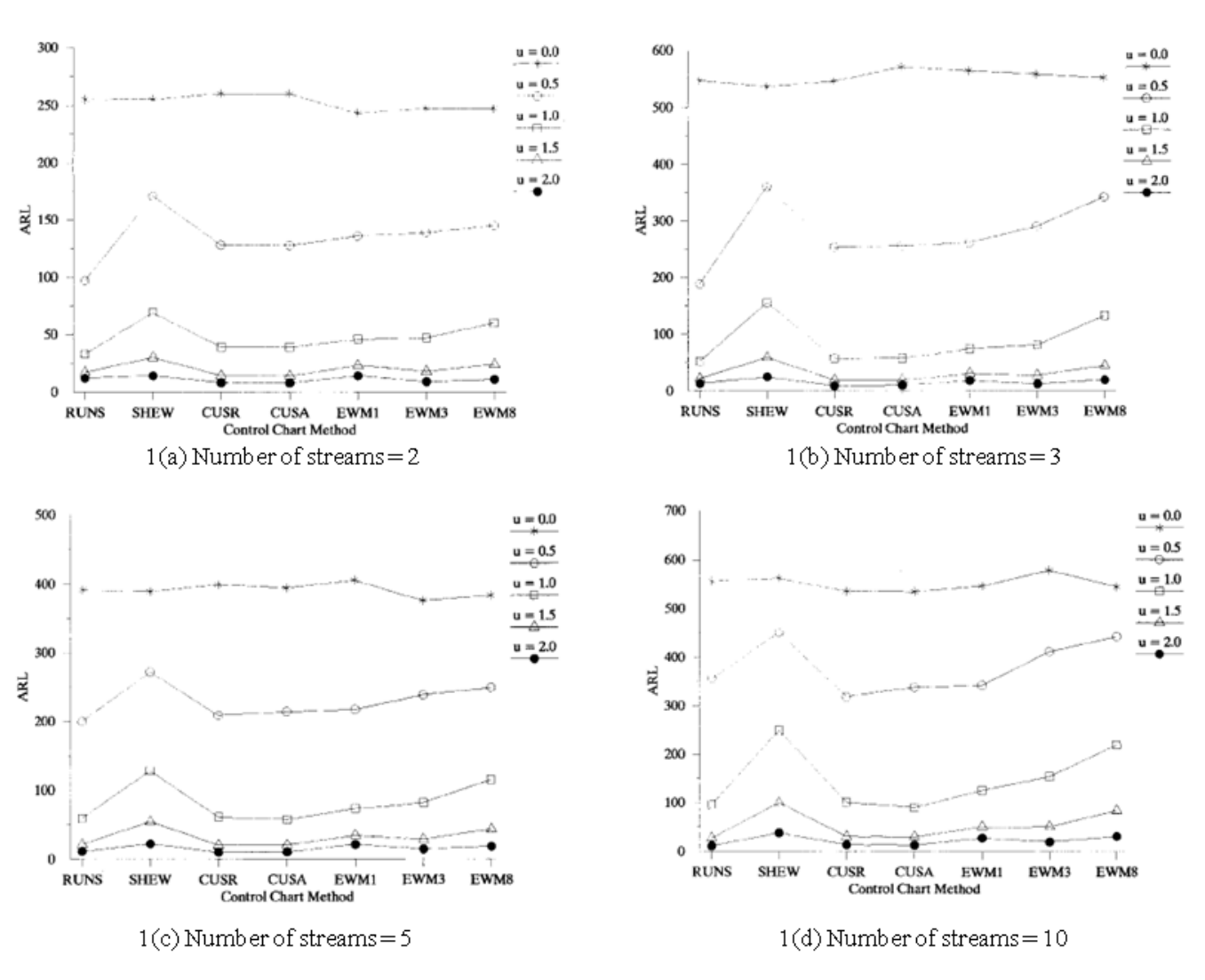

Fig. 1. Comparison of MSP control chart ARLs at various number of streams [2] (reprint with permission).

The comparison results are as follows:

- Shewhart - $R_{t}$ chart has the worst sensitivity to detect shift size less than two standard deviations. However, if the shift size is greater than two standard deviations, the sensitivity of Shewhart $-R_{t}$ chart is comparable to other charts.

- EWMA - $R_{t}$ chart has the sensitivity less than CUSUM - $R_{t}$ in every angle of comparison.

- In case the number of streams is 2-3 streams and the shift size is less than one standard deviation, GCC with run scheme is more sensitive than CUSUM - $R_{t}$. On the other hand, if shift size is more than one standard deviation, CUSUM - $R_{t}$ is more sensitive than GCC with run scheme.

- When the number of streams is equal to 5, the sensitivity of GCC and CUSUM - $R_{t}$ are comparable. 
- With 10 or more streams, the sensitivity of CUSUM - $R_{t}$ is better for shift size less than 2 standard deviations while the sensitivity of GCC with run scheme is better for shift size more than 2 standard deviations.

- In the case that there are several streams out of control, the ARL comparison between GCC with run scheme and CUSUM - $R_{t}$ is shown as Fig. 2. The RUN1, RUN2, RUN4, RUN6 representing GCC that 1, 2, 4 and 6 streams are out of control respectively and the CUS1, CUS2, CUS4, CUS6 representing CUSUM - $R_{t}$ that has 1,2, 4 and 6 streams are out of control respectively. The results of comparison show that with equal number of streams, the sensitivity of CUSUM - $R_{t}$ is better than that of GCC.
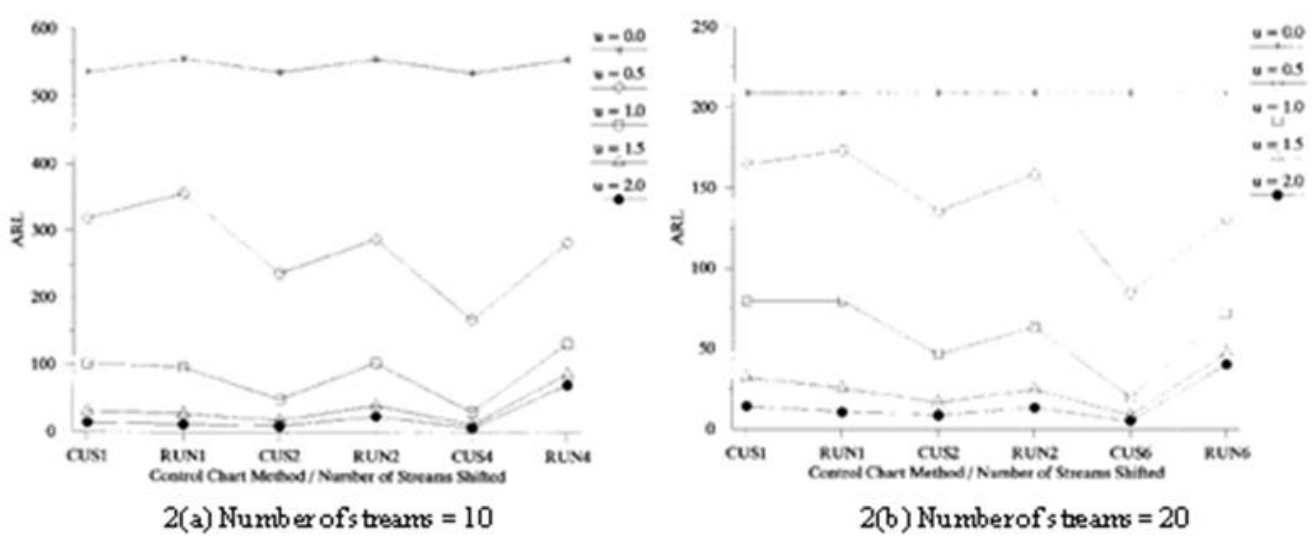

Fig 2. Comparison of MSP control chart ARLs when multiple streams shift for total streams $=10$ and 20 [2] (reprint with permission).

CUSUM - $R_{t}$ not only provide better sensitivity in detecting multiple streams shift than GCC but its control limits can also be designed to yield $\mathrm{ARL}_{0}$ close to 370 . There are two choices to design the control limits of CUSUM - $R_{t}$. First, by determining $\mathrm{k}$ value and use the simulation for determining the control limits. Second, by transforming $R_{t}$ to normal distribution then design the CUSUM transformed $R_{t}$ using the same method as CUSUM - $R_{t}$ for normal distribution.

Although $\overline{\bar{X}}$ chart and CUSUM - $R_{t}$ have more merits than GCC in many aspects, CUSUM - $R_{t}$ also has the weakness that is information gathered from this control chart is less than that of the 1 chart per stream. In addition, if the mean of each stream is significantly different and the correlation is less than 1, CUSUM - $R_{t}$ will lead to false decision on the process status [3]. Besides that, the determination of the control limits and the interpretation of CUSUM - $R_{t}$ are more difficult than other MSP control charts.

\subsection{Schewhart chart for each stream with adjusted control limits}

In case mean of each stream is significantly different and the correlation among streams is low the use of a single chart on all streams, for instances GCC or $\bar{X}-R_{t}$ chart, will lead to wrong conclusion on process status. To solve this problem, Meneces et al. [4] have proposed a Schewhart control chart for each stream. The control limit of this chart are widen from the original \pm 3 sigma limits of Schewhart control chart in order to maintain $\mathrm{ARL}_{0}$ at 370 , even though the number of streams increase.

The separated charts for each stream provide more information than a single chart for all streams. However, the number of charts is also increased as the streams increased. With resources constraint, ones may not be able to separate charts for each stream.

\section{A guideline to select control charts for multiple stream processes control}


Section 2 discusses about the situation where each MSP control chart is appropriate. The consideration is based on one or more factors, which are correlation among streams, difference among streams average, limitation to use one chart per stream, the number of streams, shift size of streams average to be detected, and the deviation of $\operatorname{ARL}(1)_{0}$ and $\operatorname{ARL}(2)_{0}$ from 370 . The result reveals that there is no outstanding MSP control chart that is better than others on all aspects. If inappropriate MSP control chart is selected, the decision upon the process status and the troubleshooting will be wrong. Therefore, the above-mentioned factors are used as the basis to establish the guideline for selecting the MSP control charts that correspond to the streams characteristics. To develop the guideline, these factors are arranged to start with the situation where simple Shewhart control chart is appropriate. Then, other factors are considered to cover the situations where more complex MSP control charts are appropriate.

Figure 1 shows the guideline to select the control charts for multiple stream processes. This guideline can be applied to wide range of applications and industries, for example, the multi head bottle-filling machine in beverage industry, multiple spindle winding machine in textile industry, multi spindle drilling machine in automotive industry and etc.

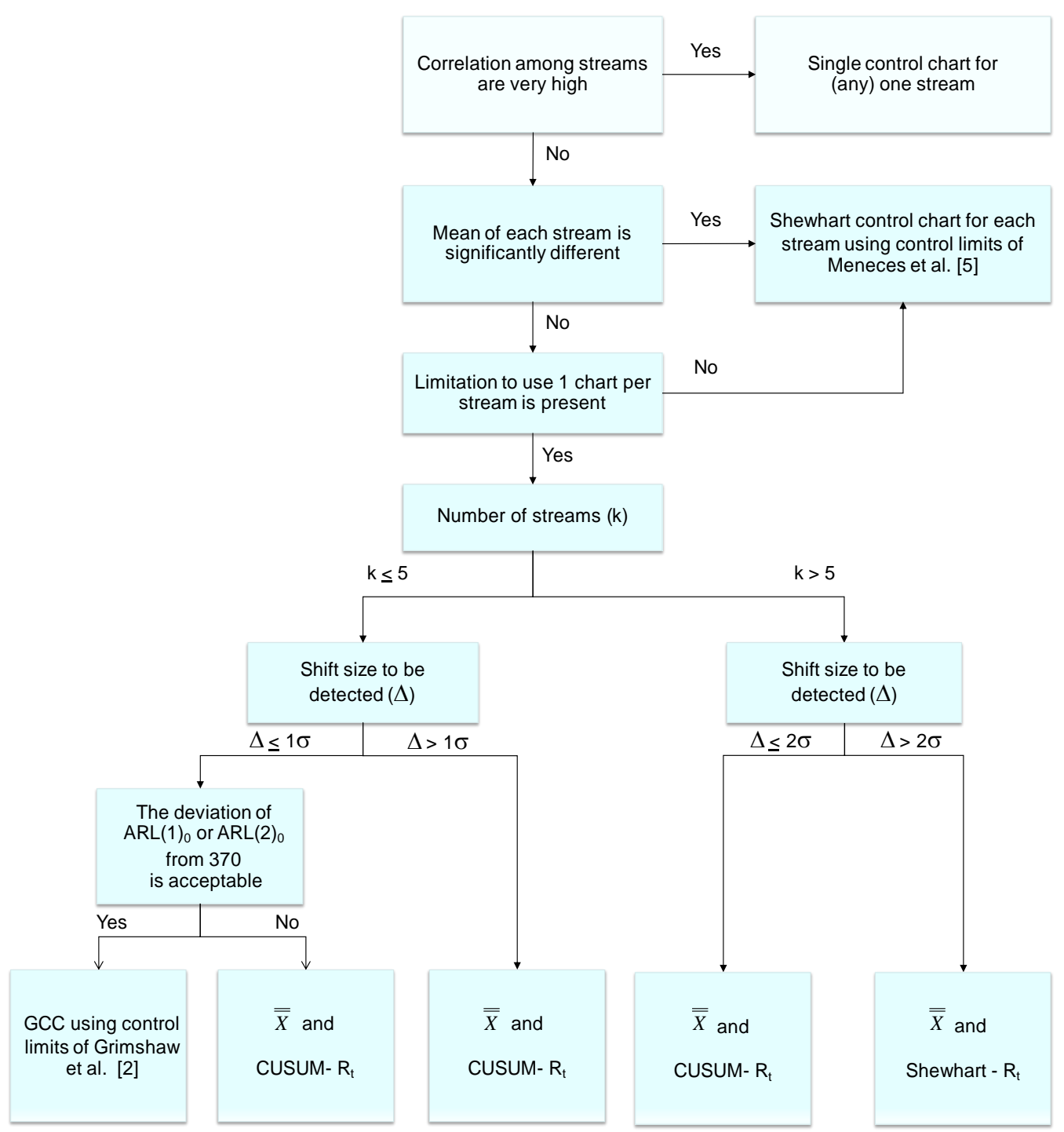

Fig. 3. A guideline to select control chart for multiple stream processes control.

From Fig. 3, if correlation among streams is very high, say, nearly perfectly correlated, the single chart for (any) one stream will represent all streams status. However, if this condition is not met, the single chart for (any) one stream cannot represent the situation of other streams correctly.

When the mean of streams are significantly different and correlation among streams is not nearly perfect, practitioner should focus on actions that reduce the variation between streams. This will result in overall process improvement. After that, select the appropriate MSP control charts that align to 
streams characteristics. However, if the improvement is not worthwhile in the economic aspect, the separated charts for each stream should be used that is the Schewhart control chart for each stream with adjusted control limits in order to maintain $\mathrm{ARL}_{0}$ close to 370, regardless of number of streams.

With information technology, nowadays the data collection, control charts plotting and alarming can be easily made with convenience. Therefore, whether there are significantly different in mean of streams or not, whenever enough resources are available and correlation among streams is not so high, practitioner should implement one chart per stream that is the Shewhart control charts for each stream because it provides more information to investigate the special cause than GCC or $\overline{\bar{X}}-R_{t}$ chart.

In case that the average of each stream is not significantly different and practitioners has the limitation to use one chart per stream, the MSP control charts will be selected based on the number of streams and the shift size of mean to be detected as follows:

- For number of streams less than or equal to 5 , if the shift size to be detected is less than or equal to 1 standard deviation and it is acceptable that the $\operatorname{ARL}(1)_{0}$ or $\operatorname{ARL}(2)_{0}$ are different from 370 , then GCC with adjusted control limits of Grimshaw et al. [3] is recommended. This because, in this case, GCC has better sensitivity than CUSUM $-R_{t}$. Moreover, the calculation of control limits and the charts interpretation is easier than CUSUM - $R_{t}$. However, if ARL $(1)_{0}$ or ARL(2) $)_{0}$ unacceptably differ from $370, \overline{\bar{X}}$ and CUSUM - $R_{t}$ charts are recommended because their control limits can be designed to closed to 370 .

- When the number of streams is less than or equal to 5 and the shift size is greater than 1 standard deviation, $\overline{\bar{X}}$ chart and CUSUM - $R_{t}$ should be used because the sensitivity is better than that of other MSP control charts.

- When the number of streams is greater than 5 and the shift size is less than or equal to 2 standard deviations, the $\overline{\bar{X}}$ and CUSUM $-R_{t}$ are recommended because the sensitivity is better than that of other charts and the control limits can be designed to achieve $\mathrm{ARL}_{0}$ close to 370 .

- When the number of streams is greater than 5 and the shift size is greater than 2 standard deviations, $\overline{\bar{X}}$ and Shewhart $-R_{t}$ should be used because its sensitivity is better than that of GCC and comparable to $\overline{\bar{X}}$ and CUSUM - $R_{t}$. The design of control limits and the interpretation of Shewhart - $R_{t}$ can be made easier than CUSUM - $R_{t}$. Besides, the control limits of Shewhart - $R_{t}$ can be designed to provide $\mathrm{ARL}_{0}$ closed to 370 .

\section{A case study}

The factory in this case study is an electronic manufacturing service (EMS) company. The main product of the factory is the optical transceiver which is one component of fiber optic telecommunication network. One of significant parameters that affect to the main function of this device is frequency of the output signal. The specification given by a customer is the difference between the output frequency and the target frequency must be within the range of $\pm 0.001 \mathrm{GHz}$. The frequency of the output signal will be adjusted by the Calibration Test machine (Fig. 4) after all components had been assembled to be the finished goods. The Calibration Test machine has 4 modules that can be operated simultaneously. By considering each module as a process stream, the Calibration Test process is a multiple streams process with 4 output streams. 3 samples were collected from each module for a period of time. The $\overline{\bar{X}}$ and $R_{t}$ charts for Calibration Test process were constructed as Fig. 5 . 


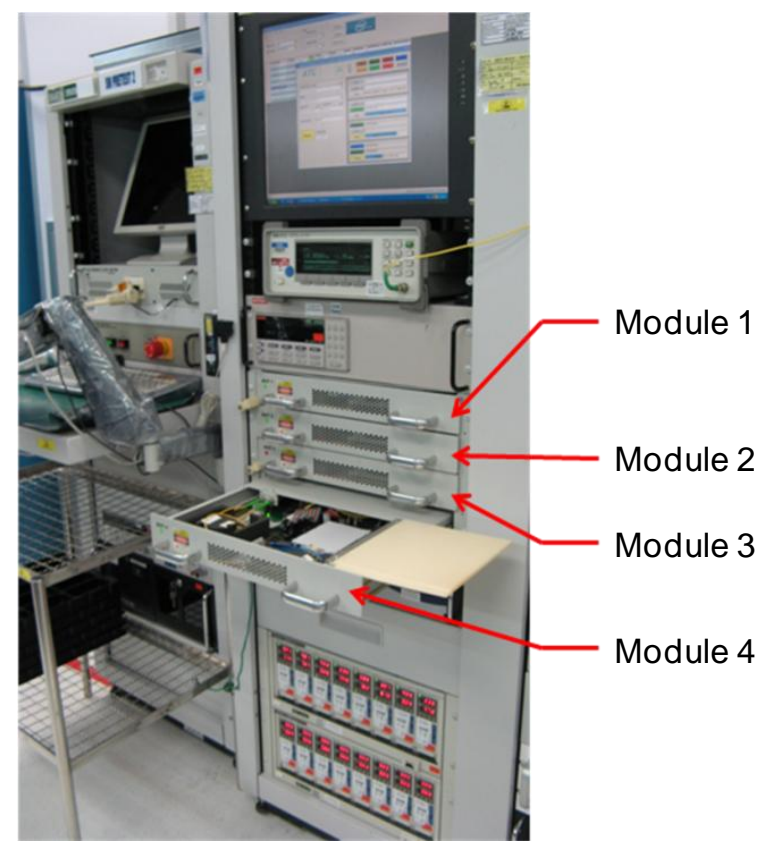

Fig. 4. Calibration Test machine.

X Bar Bar, R Chart of Calibration Test: Frequency Error

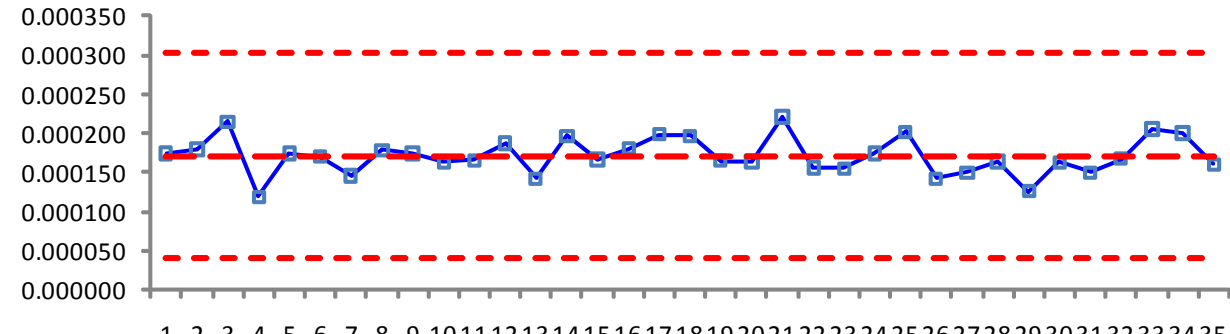

12234456778991011121314151617181920212223242526272829303132333435

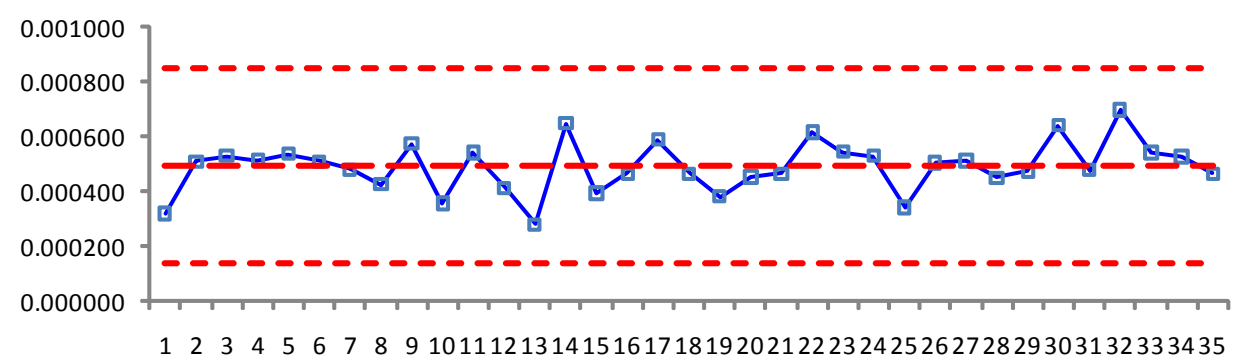

Fig. 5. $\overline{\bar{X}}-R$ Chart of Calibration Test process.

The control charts in Fig. 5 do not comply with the rational subgroup concept. It can be noticed from $\overline{\bar{X}}$ chart that all the points are closed to the center line. This is due to the control limits are widened from the significant difference between module 1 and others as shown in Fig. 6 . These controls charts are lack of sensitivity to detect the special causes in case process is out of control. 


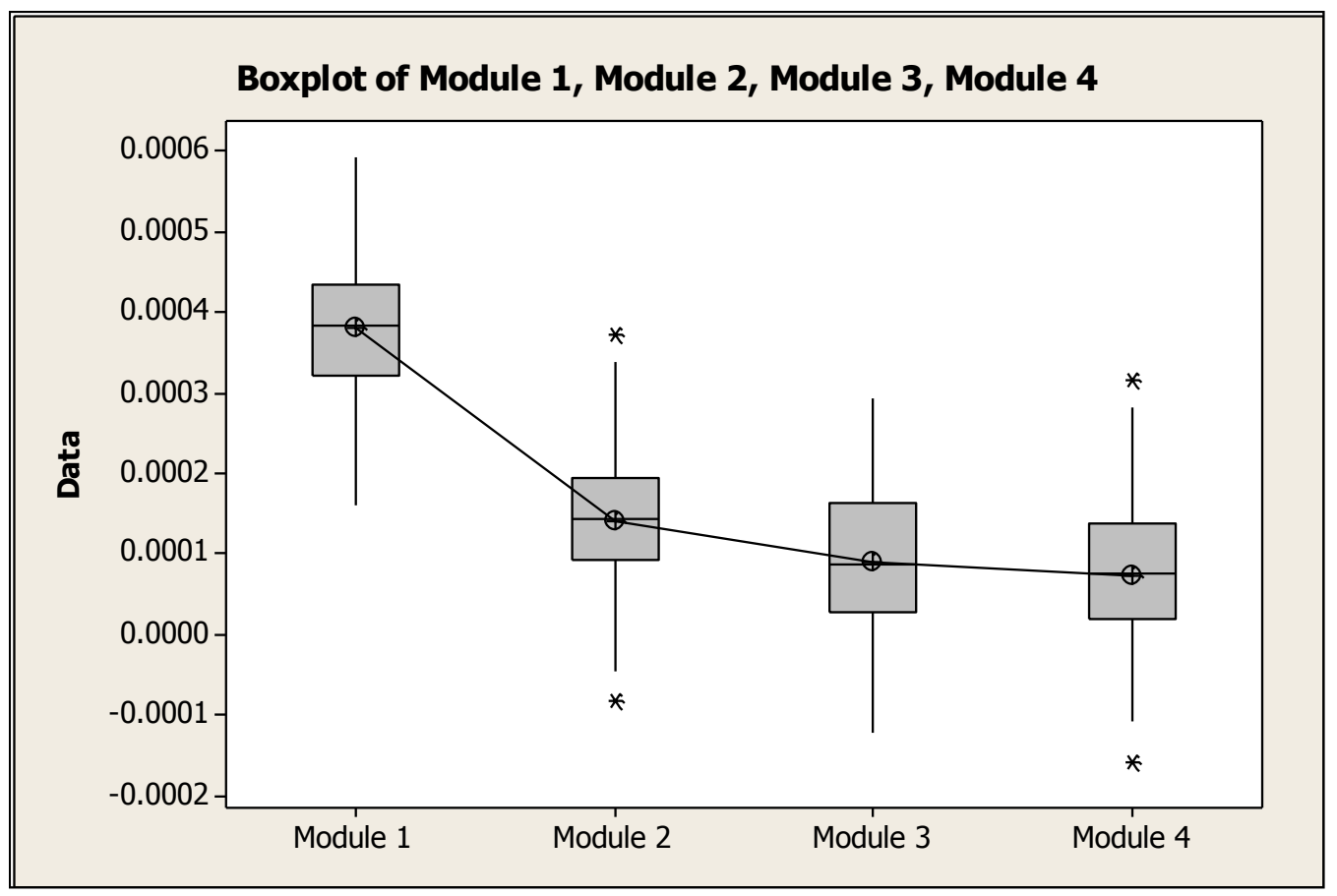

Fig. 6. Box-plot of frequency error of module 1-4 of Calibration Test machine.

Although the module 1 significantly differ from others, but in this case, the improvement action in order to close the gap is not economic merit as high expense is required and the Cpk of module 1 is high at 2.62 (Fig. 7). Although the customer accepts the difference between module 1 and others, they still require this parameter to be monitored by the control charts.

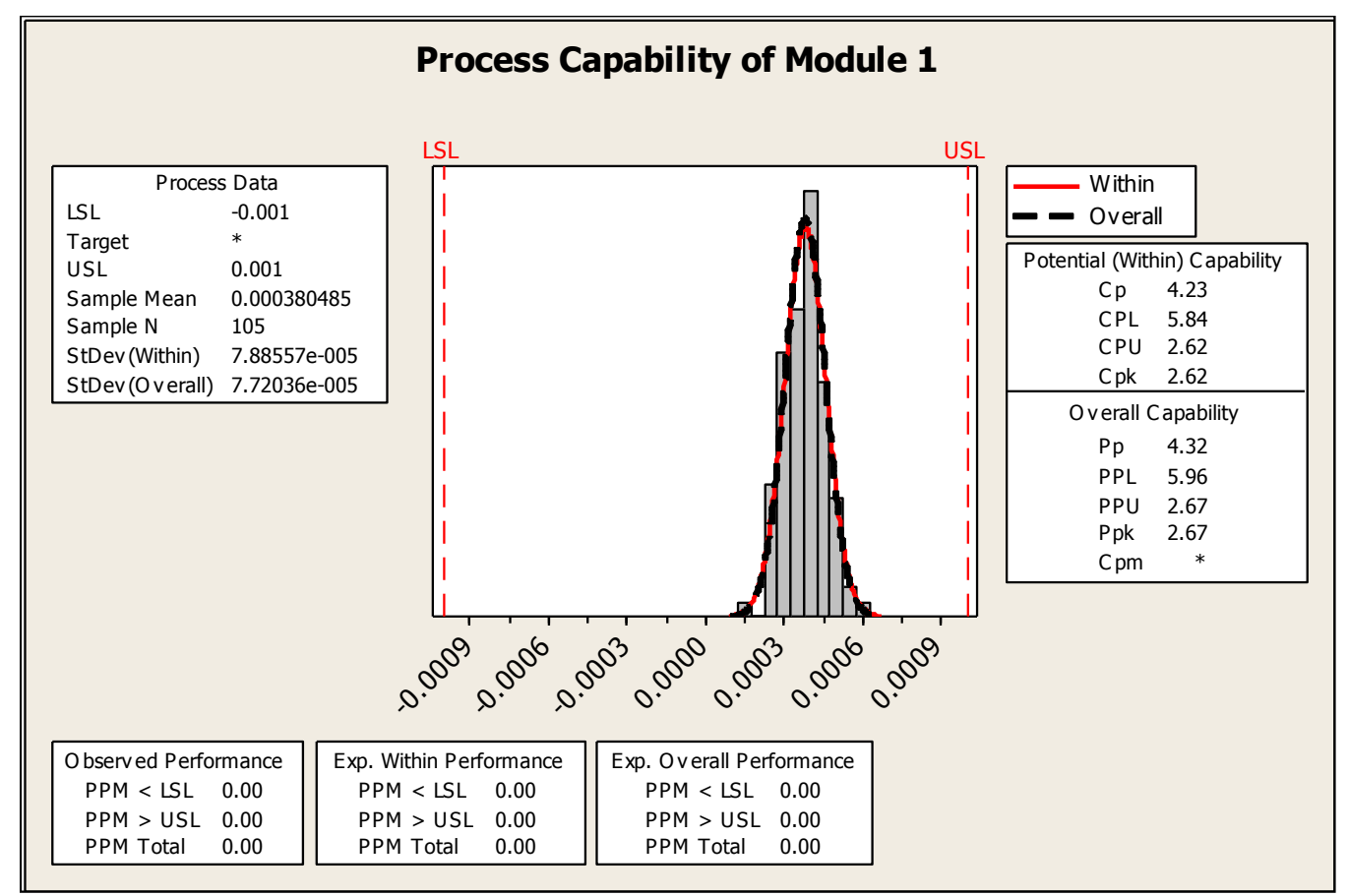

Fig. 7. Process capability of module 1.

By considering the characteristics of Calibration Test process, there is no evidence to conclude that the correlation among streams is very high. The mean between streams 1 and others are significantly different. Moreover, the factory has the online statistical process control system that makes the use of 1 
chart per 1 stream possible. Based on the guideline to select MSP control charts in Fig. 3, the suitable MSP charts for this case is the Schewhart control chart for each stream with adjusted control limits. Figure 8 demonstrates the use of Shewhart control chart for module 1-4 by using control limit of Meneces et al. [4]. These charts provide better sensitivity to detect special cause than the previous $\overline{\bar{X}}-R$ charts. Besides that, the hunting for special cause and troubleshooting can be done quicker because it can be known immediately that what streams or all streams are out of control. The 14th plotting in the range control chart of module 4 (Fig. 8(d)) is out of control which means that special cause may occur in the module 4 . Hence, the process controller must search for special cause and solve the problems by focusing on the 4th module.

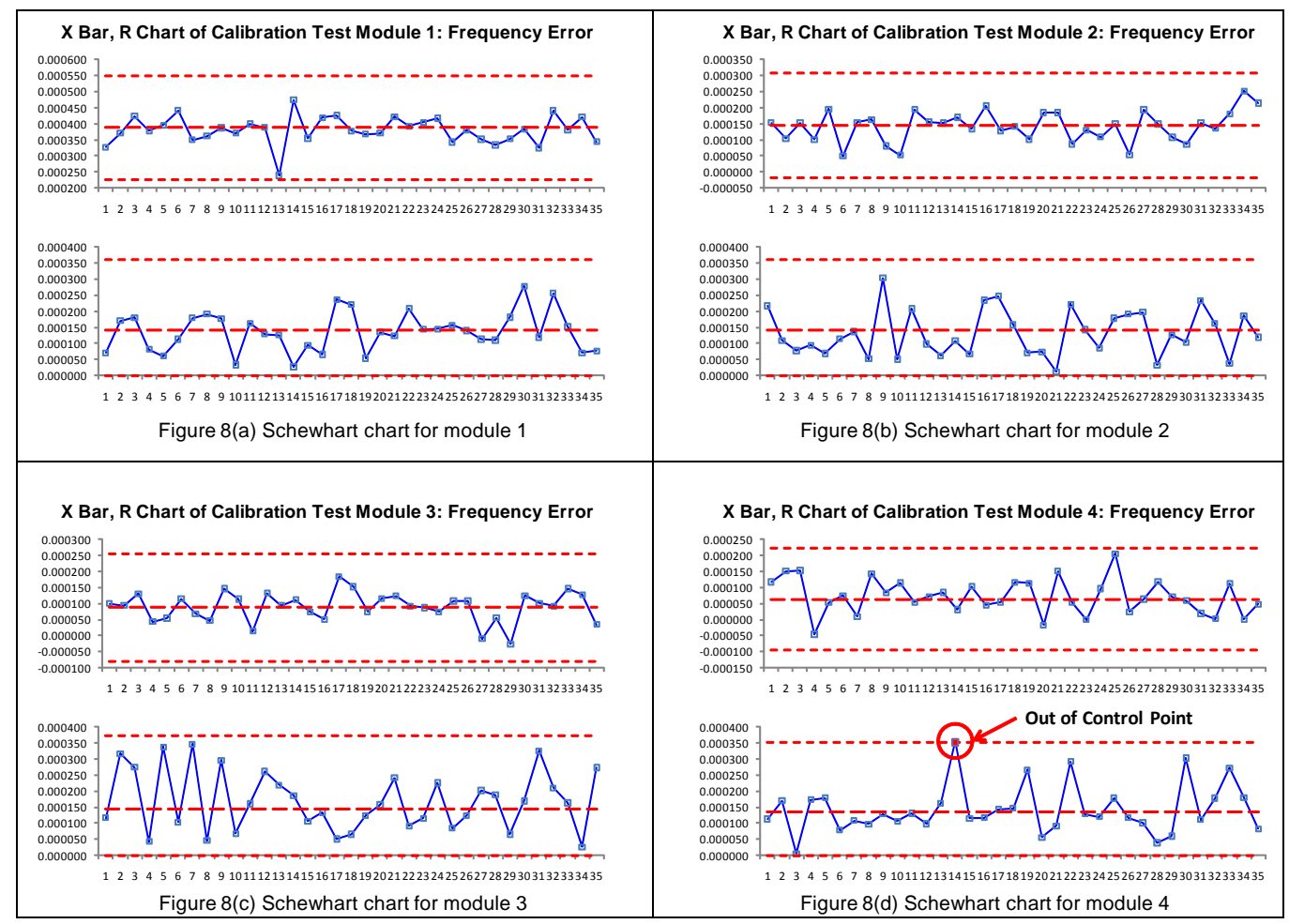

Fig. 8. Schewhart control chart for module 1-4 using control limits of Meneces et al. [1]

In the case that the mean of each stream is significantly different but has to be accepted as improvement is not worthwhile, using a single chart on all streams will lead to wrong decision. For instance, Fig. 9 shows the GCC of streams 1-4 of Calibration Test process. Consider the process out of control from a point outside the control limits and the run schemes with $r=5\left(\operatorname{ARL}(1)_{0}=341\right)$. Ones may conclude that the process is out of control because it can be noticed from the group $\bar{X}$ chart (the top chart in Fig. 9) that almost all largest points exceed the upper control limits and some smallest points are below the lower control limits. Moreover, stream 1 also yields maximum average more than 5 consecutive runs. Although these alarms are the general operation of GCC, but from the process controller's perspective the process is not out of control. Since stream 1 is allowed to have higher mean than others, it has nothing unusual that stream 1 is continuously plotted as maximum points on the group chart. Thus, the GCC is not recommended to be used. 


\section{Group Control Chart of Calibration Test: Frequency Error}

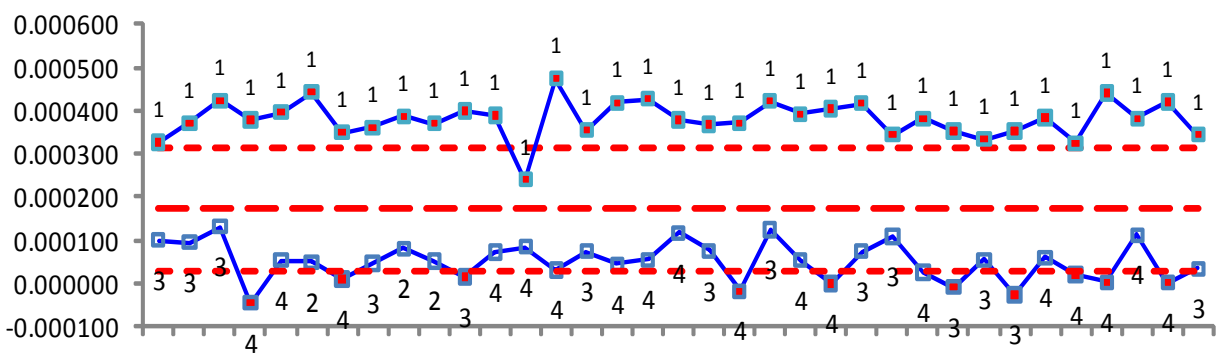

12234456678991011121314151617181920212223242526272829303132333435

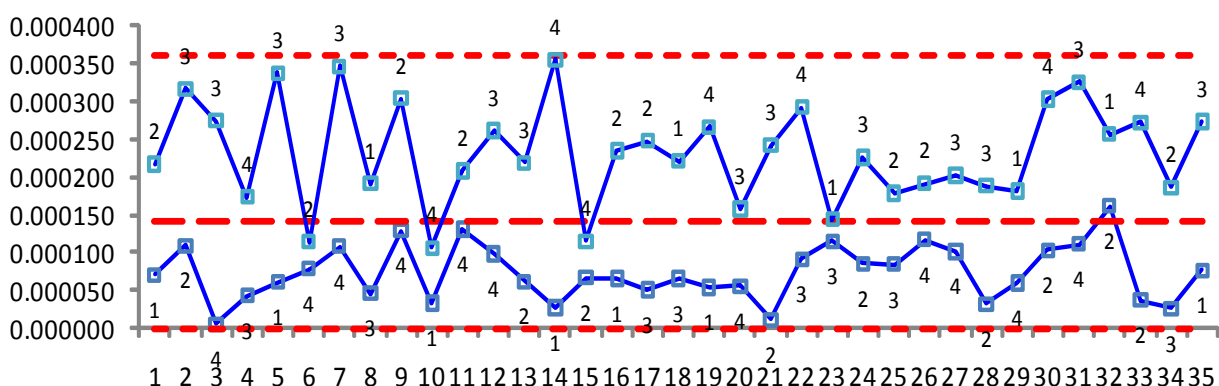

Fig. 9. Group control chart of Calibration Test process.

The $\overline{\bar{X}}$ and CUSUM - $R_{t}$ or Shewhart $-R_{t}$ are also not suitable to used. For $\overline{\bar{X}}$ and CUSUM - $R_{t}$ or Shewhart - $R_{t}$, when the mean of each stream is significantly different, the $R_{t}$ statistics will be large. The large $R_{t}$ statistics will widen the control limits of $\overline{\bar{X}}$ and Shewhart $-R_{t}$ and decrease the charts' sensitivity. Figure 10 and 11 show $\overline{\bar{X}}$ and Shewhart $-R_{t}$ charts and $\overline{\bar{X}}$ and CUSUM $-R_{t}$ charts of calibration test process respectively. The difference between mean of stream 1 and others widen the control limits of $\overline{\bar{X}}$ and Shewhart $-R_{t}$. These control limits are so wide that the charts are lack of sensitivity to detect special cause which can be noticed by all points on show $\overline{\bar{X}}$ and Shewhart $-R_{t}$ charts are plotted closely to the center line. 
X Bar Bar, Shewhart Rt Chart of Calibration Test : Frequency Error

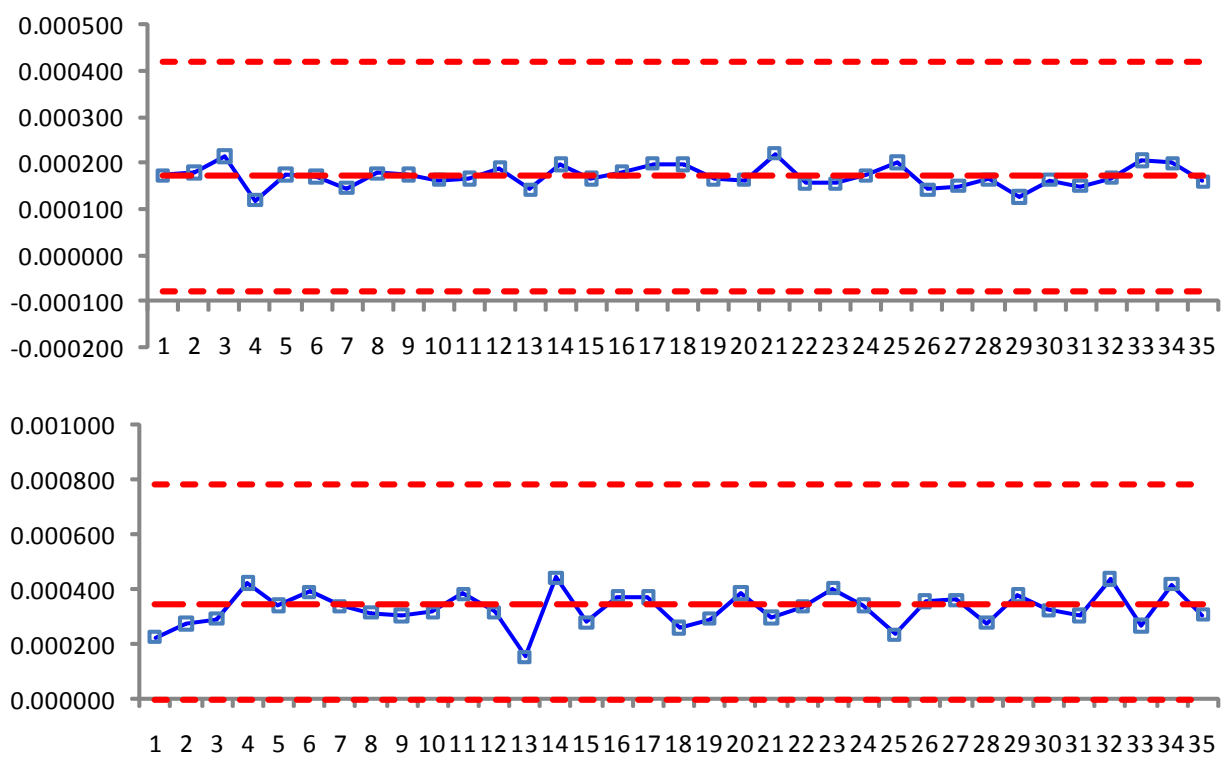

Fig. 10. $\overline{\bar{X}}$ and Shewhart $R_{t}$ charts of Calibration Test process.

\section{Bar Bar, CUSUM Rt Chart of Calibration Test : Frequency Error}

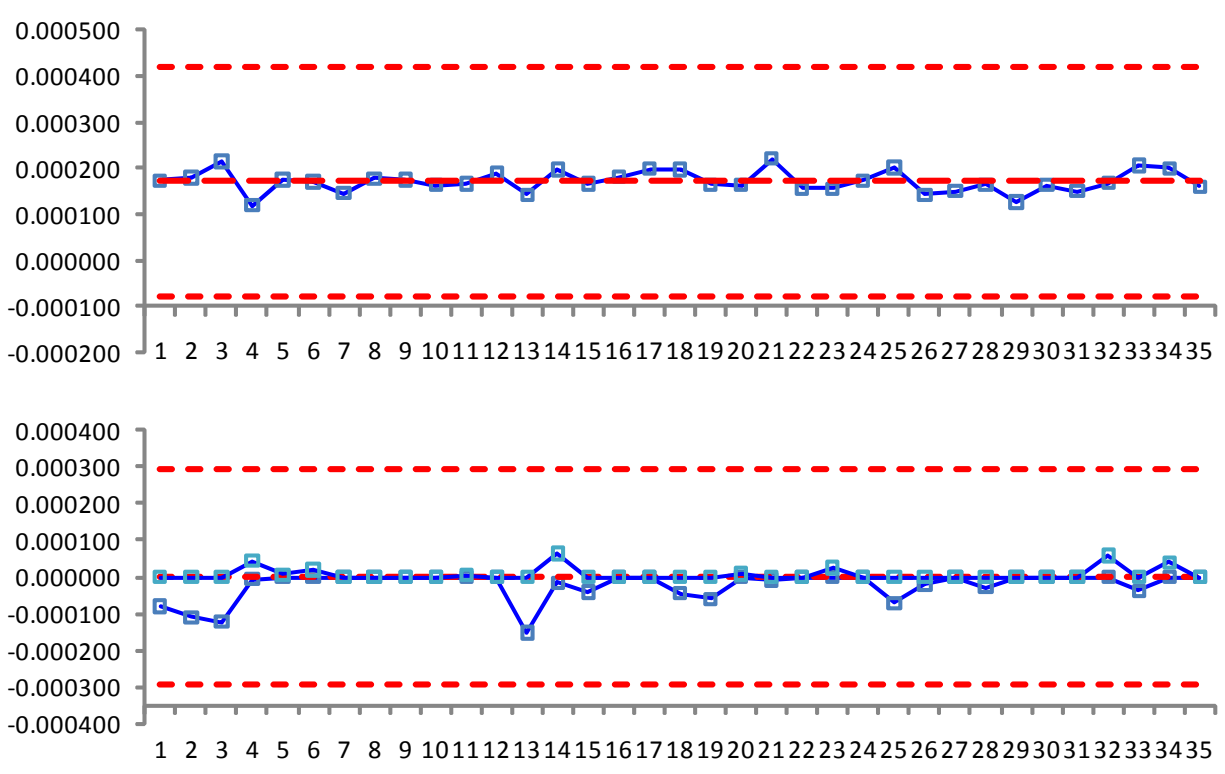

Fig. 11. $\bar{X}$ and CUSUM - $R_{t}$ charts of Calibration Test process.

\section{Conclusion}

There are many types of MSP control charts, in each type, there are strengths and weaknesses of each. There is no perfect MSP chart that is better than the others in all aspects. Thus, practitioner should select the MSP control chart corresponding to streams characteristics. If the improper MSP control chart is selected, the decisions upon process condition and action taken will have higher chance of mistakes and the use of resources in controlling the process will be inefficient.

In this paper, the guideline to select proper MSP control charts is proposed. The selection criteria are based on the following factors: number of streams, the limitation of using 1 chart to 1 stream, the difference among streams average, and the shift size to be detected. 


\section{References}

[1] D. F. Boyd, "Applying the group chart for X and R," Industrial Quality Control, vol. 7, pp. 2225, 1950.

[2] R. R. Mortell and G. C. Runger, "Statistical process control of multiple stream processes," Journal of Quality Technology, vol. 27, pp. 1-12, 1995.

[3] S. D. Grimshaw, G. R. Bryce, and D. J. Meade, "Control limits for group charts," Quality Engineering, vol. 12, pp. 177-184, 1999.

[4] N. S. Meneces, S. A. Olivera, C. D. Saccone, and J. Tessore, "Statistical control of multiplestream processes: A Shewhart control chart for each stream," Quality Engineering, vol. 20, pp. 185-194, 2008.

[5] X. Liu, R. J. MacKay, and S. H. Steiner, "Monitoring multiple stream processes," Quality Engineering, vol. 20, pp. 296-308, 2008.

[6] J. W. Lanning, D. C. Montgomery, and G. C. Runger, "Monitoring a multiple stream filling operation using fractional samples," Quality Engineering, vol. 15, pp. 183-195, 2002.

[7] D. C. Montgomery, Introduction to Statistical Quality Control, 5th ed. New York: John Wiley \& Son, 2005.

[8] L. S. Nelson, "Control chart for multiple stream processes," Journal of Quality Technology, vol. 18 , pp. 255-256, 1986. 